\title{
Induction of the hair growth phase in postnatal mice by localized transient expression of Sonic hedgehog
}

\author{
Noboru Sato, Philip L. Leopold, and Ronald G. Crystal \\ Division of Pulmonary and Critical Care Medicine, Weill Medical College of Cornell University- \\ New York Presbyterian Hospital, New York, New York 10021, USA \\ Address correspondence to: Ronald G. Crystal, Weill Medical College of Cornell University- \\ New York Presbyterian Hospital, 520 East 70th Street, ST 505, New York, New York 10021, USA. \\ Phone: (212) 746-2258; Fax: (212) 746-8383; E-mail: geneticmedicine@mail.med.cornell.edu. \\ Received for publication June 25, 1999, and accepted in revised form August 26, 1999.
}

\begin{abstract}
Hair follicles form in prenatal skin and mature in the postnatal period, establishing a growth cycle in 3 phases: telogen (resting), anagen (growth), and catagen (regression). Based on the knowledge that Sonic hedgehog $(S h b)$ expression is necessary for the embryonic development of hair follicles, and that anagen in the postnatal cycling follicle has morphologic similarities to the epithelial invagination process in embryonic skin, we hypothesized that localized, but transient, enhanced expression of the Shb gene in postnatal skin would accelerate initiation of anagen in the hair follicle cycle, with concomitant accelerated hair growth. To assess this concept, an E1- adenovirus vector, AdShh, was used to transfer the murine Shh cDNA to skin of postnatal day $19 \mathrm{C} 57 \mathrm{BL} / 6$ mice. The treated skin showed increased mRNA expression of Shh, Patched (the Shh receptor), and Gli1 (a transcription factor in the Shh pathway). In mice receiving AdShh, but not in controls, acceleration into anagen was evident, since hair follicle size and melanogenesis increased and the hair-specific keratin ghHb- 1 and the melanin synthesis-related tyrosinase mRNAs accumulated. Finally, C57BL/6 mice showed marked acceleration of the onset of new hair growth in the region of AdShh administration to skin 2 weeks after treatment, but not in control vector-treated or untreated areas. After 6 months, AdShh-treated skin showed normal hair and normal skin morphology. Together, these observations are consistent with the concept that upregulation of Shh activity in postnatal skin functions as a biologic switch that induces resting hair follicles to enter anagen with consequent hair growth.
\end{abstract}

J. Clin. Invest. 104:855-864 (1999).

\section{Introduction}

Hair follicles are the anatomic "factories" that have as their principal function the production of hair. Hair follicle neogenesis occurs in the embryo by invagination of the epidermal placode into the surrounding dermis (1-4). Postnatal folicles undergo a cycle of renewal in 3 phases: anagen (growth), catagen (regression), and telogen (resting) $(5,6)$. The first complete postnatal hair folicle cycle (first anagen, first catagen, first telogen) is completed in the first $31 / 2$ weeks after birth and is followed by the second hair cycle (second anagen, second catagen, second telogen). Despite intensive study and observed links to a number of growth factors $(7,8)$, the mechanisms underlying the biologic switch in postnatal follicles from telogen to anagen remain unknown.

Among the many genes expressed in association with the development of hair follicles in the embryo, Sonic hedgehog (Shb) appears to play a central role. Shh, one of 3 vertebrate homologues of Drosophila hedgehog $(\mathrm{Hh})$, is involved in pattern formation of vertebrate organs including brain, heart, lung, skeleton, and skin (9-20). Shh functions in association with a complex signaling pathway that includes Patched (Ptc), the Shh receptor; Smoothened (Smo), a putative transmem- brane $\mathrm{G}$ protein-coupled receptor that interacts with Ptc; Gli, a family of transcription factors; and a variety of other signaling molecules that include the Wnt and the TGF- $\beta$ families $(13,21-34)$. The relevance of Shh to hair development has been suggested by the Shh expression pattern during embryogenesis and by manipulation of Shh expression throughout embryonic development. During normal hair follicle development, Shh is expressed in follicles in the epidermal placode, and its receptor, Ptc, is detected in underlying mesenchymal condensation at approximately embryonic day 14.5 (10, 29). In adult mice, Shh is expressed in hair follicles (20), but a role for Shh in the postnatal hair follicle cycle, if any, has not been determined. Gain-of-function models in mouse, chick, and frog embryonic development show that forced expression of the Shb gene or of Shh signaling-related genes in the skin induce abnormal epidermal cell proliferation $(21,28,35-38)$. Loss-of-function models also suggest a role for Shh in embryonic hair follicle development. Fetal skin from mouse Shb homozygous mutants $\left(\mathrm{Shb}^{-/}\right)$fails to show normal epithelial invaginations during hair follicle development $(19,39)$.

Based on the data supporting a role for Shh in embryonic development of hair follicles $(19,34,36,39)$, and given that anagen during the postnatal hair follicle 


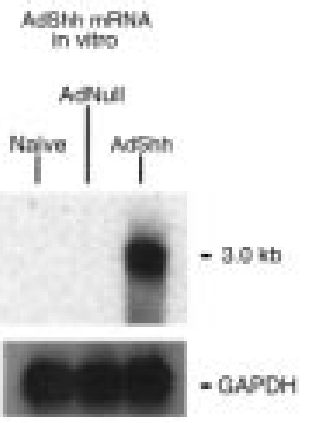

b
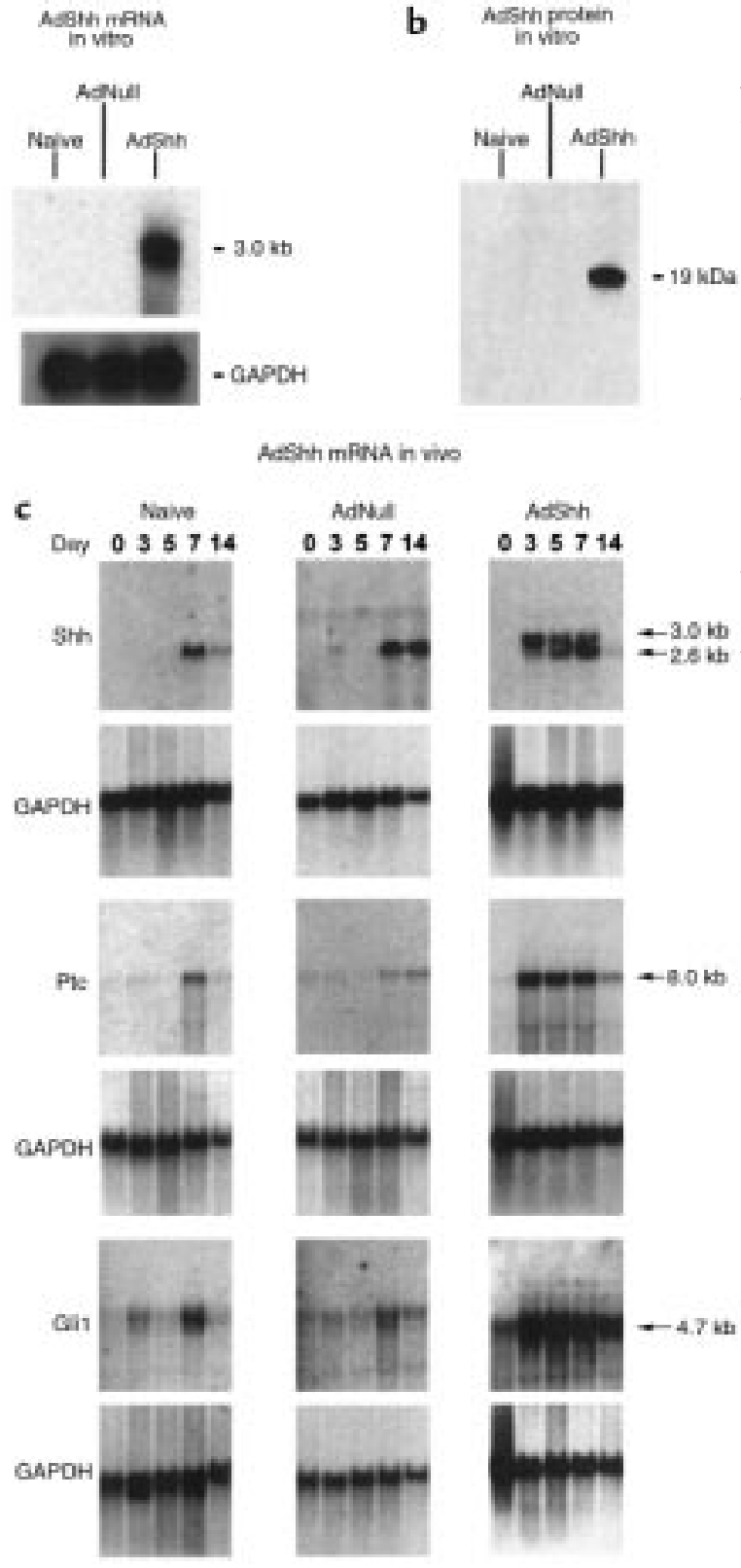

cycle shows morphologic similarities to the epithelial invagination process in embryonic skin $(1,3)$, we hypothesized that transient overexpression of Shh in postnatal skin would accelerate the initiation of anagen in the hair follicle cycle. To evaluate this concept, an adenovirus (Ad) gene-transfer vector was constructed to express Shh, with the knowledge that expression mediated by first-generation Ad vectors in immunocompetent hosts is limited to less than 2 weeks $(40,41)$, and that skin administration of Ad vectors is associated with the expression of genes in both epidermal and dermal tissues, including hair follicle epithelium (42).

\section{Methods}

Vectors. All Ad vectors used in this study were serotype 5, with deletions in the Ela, Elb, and E3 regions of the Ad genome that rendered the vectors replication-defi-

\section{Figure 1}

Functional expression of Shh by administration of AdShh in vitro and in vivo in postnatal day $19 \mathrm{C} 57 \mathrm{BL} / 6$ mice. (a) Shh mRNA after in vitro infection of A549 epithelial cells with AdShh. A549 cells $\left(10^{6}\right)$ were infected $(20$ moi) with the AdNull control vector or AdShh. After 2 days, RNA was analyzed by Northern blot with a ${ }^{32} \mathrm{P}$-labeled Shh cDNA probe. Uninfected cells (naive) and AdNull-infected cells were negative, whereas AdShhinfected cells contained a transcript of approximately $3.0 \mathrm{~kb}$ corresponding to the vector-encoded Shh transgene. Equal RNA loading was confirmed by analysis of GAPDH mRNA. (b) Shh protein expressed in vitro. Protein from A549 cells infected with AdNull or AdShh as described in a was analyzed by immunoblot for Shh protein. AdShh-infected, but not naive or AdNull-infected, A549 cells showed a 19-kDa immunoreactive band with anti-Shh. Equal protein loading was confirmed by Coomassie blue staining of an identically loaded gel (not shown). (c) Time course demonstrating expression of Shh, Ptc, and Gli1 mRNA in vivo. Dorsal skin was collected from naive C57BL/ 6 mice or $\mathrm{C} 57 \mathrm{BL} / 6$ mice at postnatal days 19, 22, 24, 26, and 33 after intradermal injection on day 19 with AdNull or AdShh ( $10^{8} \mathrm{PFU}$ for either vector). Skin was analyzed for Shh, Ptc, or Gli1 mRNA by Northern analysis on experimental days $0,3,5,7$, and 14. Expression of the 3.0-kb mRNA vector-encoded transcript (upper arrow) and the 2.6-kb endogenous Shh transcript (lower arrows) was detected in AdShh-injected mice, but the vector-derived transcript temporally preceded the endogenous transcript. Marked upregulation of Ptc and Gli1 gene expression was detected in AdShh-injected mice on experimental days 3 and 5 , when low levels of expression of these genes were seen in naive and AdNull-treated mice. Equal RNA loading was confirmed by analysis of GAPDH mRNA

cient (43). An expression cassette was inserted in the E1 position that consisted of a promoter, splicing signals, transgene, and polyadenylation sites $(43,44)$. The vectors were created and propagated in human embryonic kidney 293 cells. They were then purified through 2 $\mathrm{CsCl}$ gradients, characterized, and stored at $-70^{\circ} \mathrm{C}(43)$. Each preparation was characterized with respect to particle concentration, titer (plaque-forming units [PFU]), transgene expression, and presence of contaminating wild-type Ad (replication-competent Ad [RCA]). For acceptable preparations, the particle/PFU ratio was less than 100, and the RCA was less than 1 PFU per total dose administered $(43,45)$. The Ad $\beta$ gal vector uses a cytomegalovirus early/intermediate promoter enhancer to drive the Escherichia coli $\beta$-galactosidase ( $\beta$ gal) reporter gene (44). The AdNull control vector is identical but contains no transgene (44). The AdShh vector is similar to Adßgal but contains the murine Shh cDNA (12) in place of $\beta g a l$ cDNA. After purification on $\mathrm{CsCl}$ gradients, vectors were dialyzed into a solution of $150 \mathrm{mM} \mathrm{NaCl}, 10 \mathrm{mM} \mathrm{MgCl}, 10 \mathrm{mM}$ Tris- $\mathrm{HCl}(\mathrm{pH}$ 7.8 ), and $3 \%$ sucrose, and were stored at $-70^{\circ} \mathrm{C}$.

Ad vector administration. AdShh $\left(10^{8} \mathrm{PFU}, 20 \mu \mathrm{L}\right)$ was administered to C57BL/6 mice weighing 6.8-7.2 g on postnatal day 19. Intradermal injections were made into unclipped dorsomedial skin along the cephalocaudal axis during the transitional catagen/telogen phase (as judged by a change in skin color from gray to pink) (46). The injection, angled toward the head, produced a $1-\mathrm{cm}$ wheal. Mice were selected by weight for this study for 2 purposes. First, by narrowing the weight range of the mice, a more temporally synchro- 
nized transition of first telogen to second anagen was observed in the experimental population. Second, by using relatively small mice (mice weighing less than 7.2 g on day 19 included approximately $40 \%$ of the population), the dates of transition among stages of the hair cycle tended toward the later dates compared with the normal population distribution. By histologic examination on postnatal day 22 (experimental day 3 ), hair follicle morphology was determined to be typical of telogen (data not shown), in agreement with published reports (47). Therefore, injection of mice on day 19 corresponded to injection during the first catagen/first telogen transition or early telogen. For studies of AdShh injection at second telogen, 8-week-old mice were used.

RNA analysis. Northern blotting was carried out with samples from postnatal days 19, 22, 24, 26, or 33 (experimental days $0,3,5,7$, or 14 ) by purifying total RNA from dorsal skin taken from the area of injection $(1 \mathrm{~cm} \times 1 \mathrm{~cm})$ using the Trizol RNA preparation kit from Life Technologies Inc. (Gaithersburg, Maryland, USA). Isolated RNA (10 $\mu \mathrm{g} /$ lane) was separated on a $1 \%$ agarose gel, transferred to a nylon membrane (Strata-
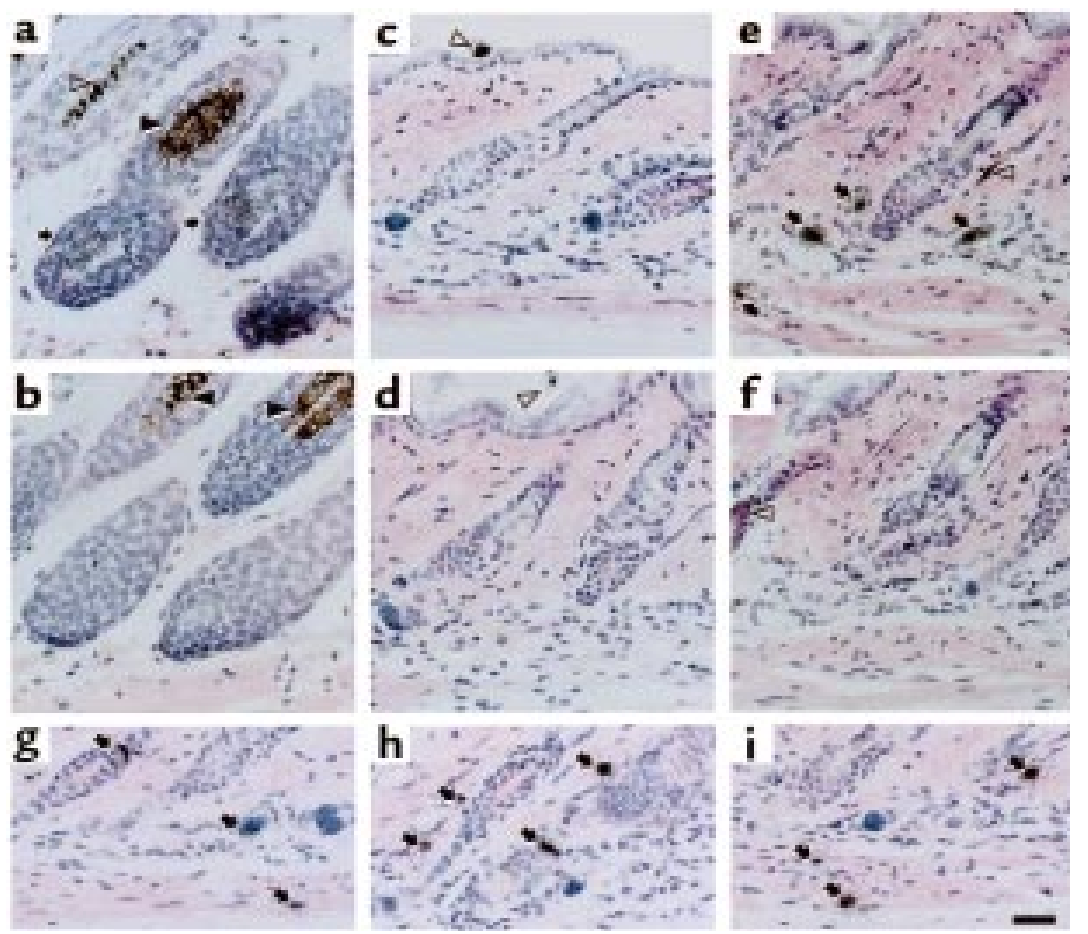

gene, La Jolla, California, USA), and hybridized with $\left[{ }^{33} \mathrm{P}\right] \mathrm{dCTP}$-labeled probes that were synthesized from cDNA by random hexamer priming (Stratagene). Hybridization conditions were optimized for each probe. After detection of target RNA, filters were stripped with $0.1 \times \mathrm{SSC}$ at $95^{\circ} \mathrm{C}$, and then reprobed with $\left[{ }^{32} \mathrm{P}\right] \mathrm{dCTP}$-labeled probe against GAPDH to ensure equal RNA loading and as a control for expression of a constitutive gene.

Protein analysis. Immunoblots were performed on AdShh-infected A549 cells scraped into PBS containing protease inhibitors [4-(2-aminoethyl)-benzene-sulfonyl fluoride, pepstatin A, trans-epoxysuccinyl-L-leucylamido(4-guanidino)butane, bestatin, leupeptin, and aprotinin]. Cells were lysed by addition of Laemmli sample buffer, sheared by passage through a 26-gauge needle, and analyzed by SDS-PAGE (15\% polyacrylamide). Proteins were transferred to a nitrocellulose membrane (Bio-Rad Laboratories Inc., Hercules, California, USA) and were detected using primary polyclonal antibodies (goat anti-Shh N-19; Santa Cruz Biotechnology Inc., Santa Cruz, California, USA) and chemiluminescent detection reagents (ECL Detection System; Amersham Life Sciences Inc., Arlington Heights, Illinois, USA).

In situ bybridization. Sites of Shh transcript expression were identified using in situ hybridization with $\left[{ }^{33} \mathrm{P}\right]$ UTP-labeled riboprobes (48-50). An antisense DNA template was generated by EcoRI digestion of a Bluescript plasmid (Stratagene) containing the full-length Shh cDNA. A sense DNA template was created by XhoI digestion of the same plasmid. Antisense riboprobes were synthesized by in vitro transcription using T7 RNA polymerase (Boehringer Mannheim Biochemicals Inc., Indianapolis, Indiana, USA). Sense probes were synthesized using T3 RNA polymerase (Boehringer Mannheim Biochemicals Inc.) in the presence of [ ${ }^{33}$ P]UTP (NEN Life Science Products Inc., Boston, Massachusetts, USA). Probe length was attenuated by alkaline hydrolysis in 100

\section{Figure 2}

Localization of Shh expression in skin by in situ hybridization before and after administration of AdShh. Paraffin sections of mouse skin during its natural anagen period (postnatal day 33) compared with postnatal day 22 naive mouse skin or skin of mice injected on postnatal day 19 with PBS, AdNull, or AdShh. The sections were analyzed by in situ hybridization using [33P] UTP-labeled antisense and sense Shh riboprobes. After hybridization, sites of probe binding were identified using a photographic emulsion, and tissue was stained with hematoxylin and eosin. Arrows indicate positive staining for Shh mRNA. Closed arrowheads indicate melanosomes in the hair follicle. Open arrowheads indicate melanin in hair shafts. (a) Anagen skin from a naive 33-day-old mouse (positive control) hybridized with an antisense Shh complementary to Shh mRNA. (b) Adjacent tissue section of anagen skin hybridized with a sense Shh probe. (c) Naive postnatal day 22 skin hybridized with an antisense Shh probe. (d) Postnatal day 22 skin 3 days after injection with $10^{8}$ PFU of AdNull hybridized with an antisense Shh probe. (e) Postnatal day 22 skin 3 days after injection of $10^{8}$ PFU of AdShh hybridized with an antisense Shh probe. (f) Postnatal day 22 skin 3 days after injection with $10^{8} \mathrm{PFU}$ of AdShh hybridized with a sense Shh probe. (g-i) High-magnification examples of cells in AdShh-injected mouse skin hybridized with antisense Shh probe. Scale bar: $50 \mu \mathrm{m}$. 

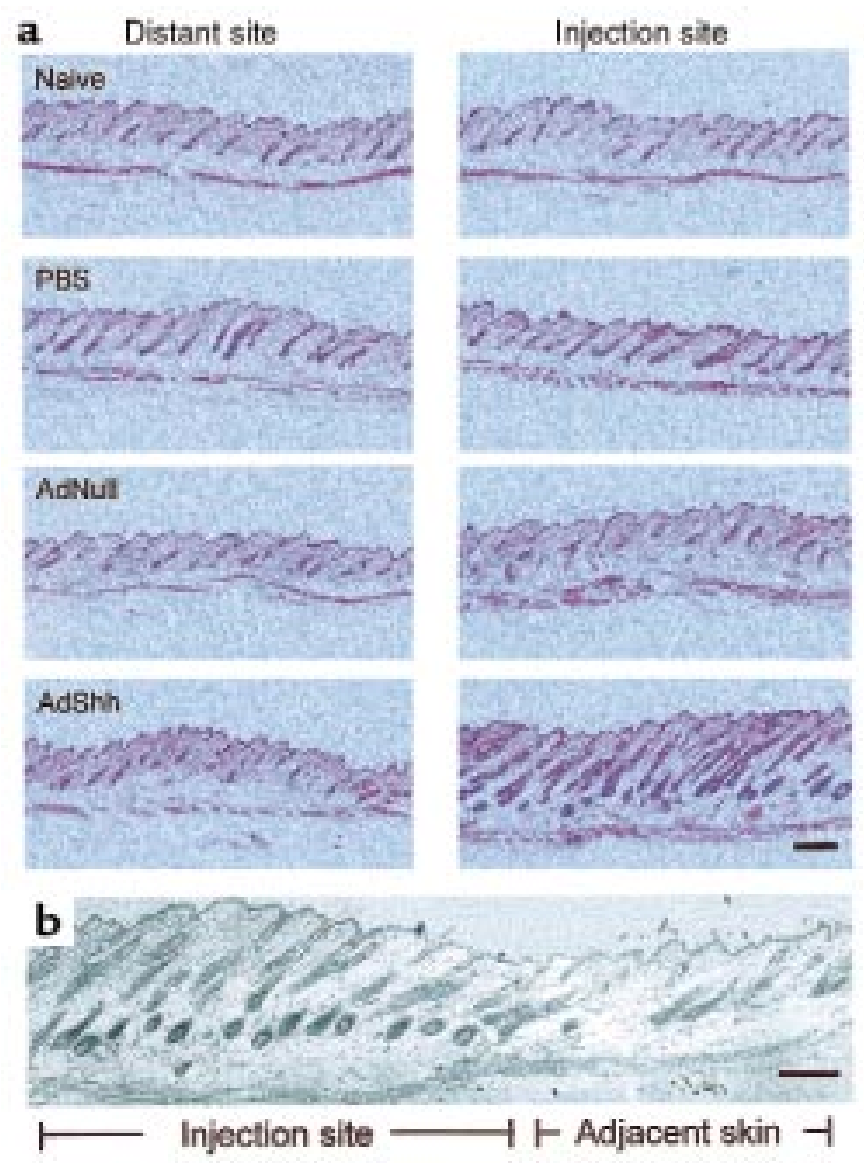

d
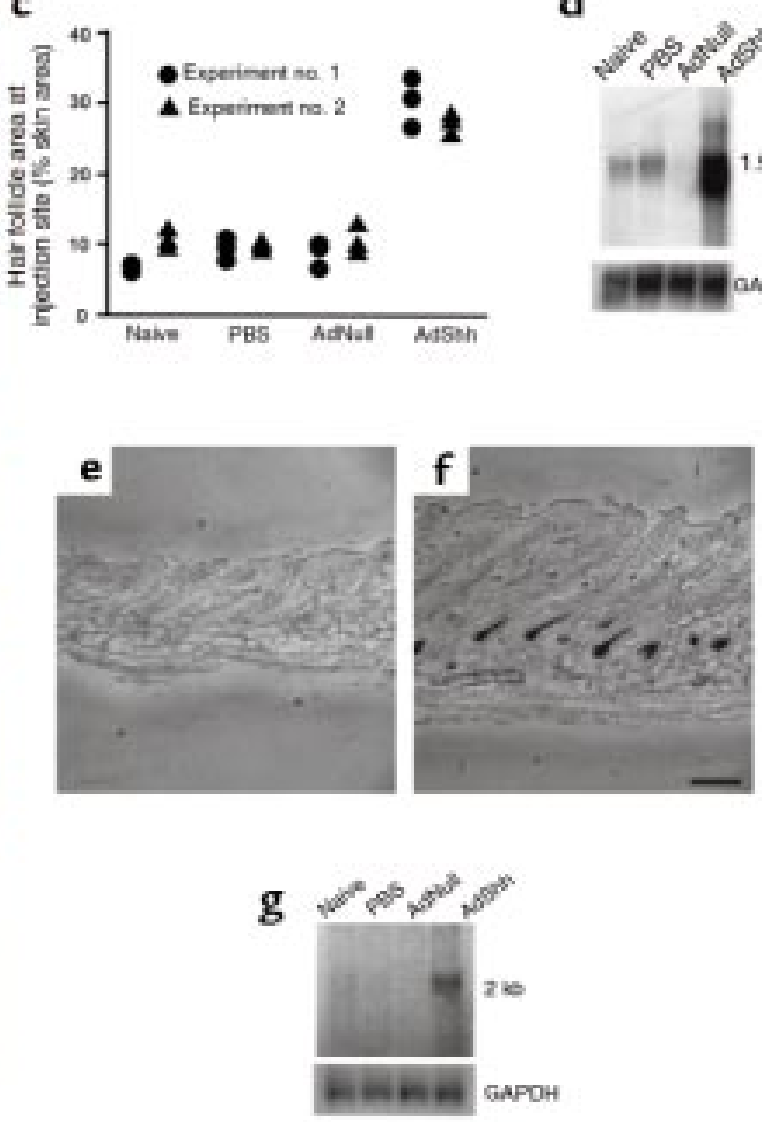

Figure 3

Induction of hair follicle growth and melanogenesis after intradermal administration of AdShh. AdShh, AdNull, or PBS was administered to the dorsal skin of postnatal day 19 C57BL/ 6 mice as in Figure 1c, and analyses were performed on postnatal day 26. (a) Histologic evaluation 7 days after vector administration. The AdShh-injected skin has increased depth of the dermal layer, increased follicle length, and increased follicle area compared with the naive, PBS, and AdNull controls. The changes associated with AdShh injection were limited to the area of injection (injection site vs. distant site [control]). (b) Histologic evaluation of the border of the injection site 7 days after vector administration. Hair follicles in the injection site are in late anagen; hair follicles in adjacent skin are in early anagen. A corresponding change in skin thickness was noted. (c) Quantitation of follicle area as a percentage of the total dermal/epidermal area using digital imaging and pixel area integration. Each data point represents an area measurement from a representative field. Data from 3 fields are shown per mouse; data from 2 mice are shown per treatment. (d) Northern analysis showing enhanced expression of the hair-specific keratin ghHb-1 gene induced by AdShh in vivo relative to the naive, PBS, and AdNull controls. Equal RNA loading shown by probing for GAPDH mRNA. (e and f) Melanin expression in hair follicle. Accumulation of melanin was evaluated by bright-field microscopy of unstained paraffin sections. Skin at the site of injection of AdShh (f), but not AdNull-injected skin (e), showed increased melanin in hair follicle bulbs. Skin of PBS-injected and naive control mice was similar to skin of AdNull-injected mice (not shown). (g) Northern analysis showing upregulation of melanogenesis-related tyrosinase gene expression after administration of AdShh relative to the naive, PBS, and AdNull controls. Equal RNA loading was confirmed by probing for GAPDH mRNA. Scale bars: $200 \mu \mathrm{m}$.

$\mathrm{mM}$ carbonate buffer ( $\mathrm{pH} 10.2$ ) for 30 minutes at $60^{\circ} \mathrm{C}$ before hybridization. After prehybridization for 3 hours at $65^{\circ} \mathrm{C}$, sections were hybridized with probes for 16 hours at $65^{\circ} \mathrm{C}$, and then washed, treated with RNase, dehydrated, and coated with photographic emulsion. After 3 weeks, slides were developed and stained with hematoxylin and eosin.

Histologic analysis. Transverse paraffin sections $(5 \mu \mathrm{m}$ thick) from mouse skin were fixed by immersion in $4 \%$ paraformaldehyde, and then stained with hematoxylin and eosin. Quantitative determination of hair follicle area on these sections was conducted using digital bright-field imaging. Images were collected with a Nikon Microphot SA microscope with a 10× Plan DIC objective, and a cooled CCD camera $(512 \times 512$ pixel, 1 MHz, back-illuminated, with a UV-VIS-coated chip; Princeton Instruments Inc., Trenton, New Jersey, USA), and were displayed using quantitative image analysis software (MetaMorph; Universal Imaging Corp., West Chester, Pennsylvania, USA). Follicle area and total area were determined by free-form outlining of regions on digital images and integration of regions based on pixel area. Three fields at the injection site were analyzed for each group (naive, PBS-injected, AdNull-injected, and AdShh-injected). Melanogenesis was evaluated in unstained paraffin sections.

Scanning electron microscopy. Hair shafts were collected from normal C57BL/ 6 mice or from the site of AdShh 
injection 14 days after vector administration. Hairs were mounted on aluminum stubs with double-sided tape, coated with gold platinum (Electron Microscopy Sciences, Ft. Washington, Pennsylvania, USA) using a PELCO 91000 Sputter Coater (Ted Pella, Redding, California, USA), and then viewed at $\times 3000$ using a JEOL100CX electron microscope with an ASID scanning attachment (JEOL, USA, Peabody, Massachusetts, USA) operating at $20 \mathrm{kV}$.

Assessment of $\beta$ gal transgene expression. The $\beta$ gal reporter gene was assessed in tissue homogenate in a luminometer using a Galacto-Light kit (Tropix Inc., Bedford, Massachusetts, USA), and adjusted for total protein using the BCA assay from Bio-Rad Laboratories Inc. The limit of detection was 1,000 relative light units/mg protein.

\section{Results}

Shb expression. Several lines of evidence demonstrated that administration of AdShh led to expression of the Shb coding sequences, resulting in production of a functional Shh protein. First, Northern analysis of A549 cells infected with the Ad vector encoding murine Shh demonstrated transcripts of approximately $3.0 \mathrm{~kb}$, corresponding to the sum of the full-length Shh cDNA (2.6 $\mathrm{kb})$ with associated vector-derived $5^{\prime}$ - and $3^{\prime}$-untranslated regions ( $400 \mathrm{bp}$; Figure 1a). In contrast, Northern analysis of naive A549 cells or A549 cells infected with AdNull demonstrated no Shh transcripts. Second, immunoblot analysis demonstrated that the AdShhinfected A549 cells produced a $19 \mathrm{kDa}$ processed form of the murine Shh protein (Figure 1b). Third, Shh expression directed by AdShh could also be detected in vivo by Northern analysis. In naive C57BL/6 mouse skin, upregulation of the endogenous $2.6-\mathrm{kb}$ Shb gene expression was observed at experimental day 7 when mouse skin entered early anagen (Figure 1c). By day 14, endogenous $S h b$ gene expression was diminished. C57BL/6 mouse skin injected with AdNull showed a pattern of Shh expression similar to that seen in naive skin. In contrast, AdShh-injected mouse skin showed strong Shb gene expression at day 3 after injection (the AdShh-encoded transcript is $3.0 \mathrm{~kb}$, larger than the 2.6$\mathrm{kb}$ endogenous Shh seen in the controls at day 7). At day 3 and more clearly at day 5, 2 distinct bands were observed in close proximity. Vector-encoded message appeared at $3.0 \mathrm{~kb}$. The lower band at $2.6 \mathrm{~kb}$ corresponded to endogenous Shh transcript that had the same size as that seen in the nontreated mouse skin. At day 7 , endogenous signal exceeded vector-encoded signal, and at day 14 the upper band derived from AdShh could no longer be detected. These results raise the possibility that exogenous Shh expression derived from AdShh induced the endogenous Shh expression as early as day 3 after injection (postnatal day 22), when naive skin showed no endogenous Shh mRNA expression.

Finally, in addition to Shh, the AdShh vector induced in vivo expression of mRNA transcripts of both Ptc and Gli1, two of the mediators that act as downstream effec-
Table 1

AdShh-mediated acceleration of hair follicle maturation

\begin{tabular}{|c|c|c|c|}
\hline \multicolumn{4}{|c|}{ Number of positive mice/total number of mice evaluated } \\
\hline Treatment & $\begin{array}{l}\text { Hair follicles } \\
\text { area }(\text { day } 7)^{\mathrm{A}}\end{array}$ & $\begin{array}{c}\text { Melanogenesis } \\
\quad(\text { day } 7)^{\mathrm{B}}\end{array}$ & $\begin{array}{l}\text { Hair growth } \\
(\text { day } 14)^{\mathrm{C}}\end{array}$ \\
\hline Naive & $0 / 5$ & $0 / 5$ & $0 / 5$ \\
\hline PBS & $0 / 5$ & $0 / 5$ & $0 / 5$ \\
\hline AdNull & $0 / 8$ & $0 / 5$ & $0 / 5$ \\
\hline AdShh & $7 / 7$ & $6 / 7$ & $6 / 7$ \\
\hline
\end{tabular}

ASee Figure 3a ${ }^{B}$ See Figure 4, a and b. CSee Figure 4, c and d.

tors in the Shh signaling pathway $(2,13,23)$. Naive and AdNull-injected skin showed upregulation of both Ptc and Gli1 transcripts on experimental day 7 and day 14, on which days endogenous Shh was also upregulated, suggesting that the Shh signaling pathway is normally activated during the hair cycle. In contrast, AdShhinjected skin showed remarkable upregulation of both Ptc and Gli1 gene expression on experimental days 3, 5, and 7, parallel to the Shb expression pattern. Administration of the control AdNull vector appeared to shift expression of endogenous Shh, Ptc, and Gli1 from predominantly day 7 to expression on both days 7 and 14 .

In situ bybridization. To establish the localization of Shh expression on day 3 after administration of AdShh, paraffin-embedded sections were hybridized with either antisense or sense [33P]UTP riboprobes, and were evaluated using a photographic emulsion. As a positive control, anagen skin was evaluated for Shh transcripts. In anagen skin, Shh expression was limited to the matrix of the hair follicle bulb and was often observed on the anterior side of the hair follicle (Figure 2 , a and $b$ ), in agreement with previous reports in postnatal skin (34). Naive telogen skin (postnatal day 22), as well as skin injected with AdNull, showed no detectable Shh transcript using the sense probe (Figure 2, c and d). In contrast, AdShh-injected skin showed intense Shh transcript expression with antisense, but not sense riboprobes, in diverse cell types including keratinocytes in hair follicles, mesenchymal cells in dermal papillae, fibroblasts in the dermis and the subdermal fascia, and muscle (Figure 2, e-i).

Hair growth. Hallmarks of anagen include thickening of the dermal and epidermal layers of the skin, increased size of hair follicles (and thus increased area occupied by follicles in a histologic section), extension of follicles deep into the dermal adipose tissue, and initiation of melanin synthesis $(1,51)$. Histology of mouse dorsal skin was evaluated in telogen/early anagen (7 days after intradermal injection of AdShh into postnatal day 19 catagen/telogen mouse skin) at the site of injection, and in dorsal skin at a site distant from the site of injection. As controls, naive, PBSinjected, and AdNull-injected skin were analyzed. Compared with control skin, both the dermis and epidermis of AdShh-injected skin showed characteristics of anagen, including thickening, hair follicles that were 


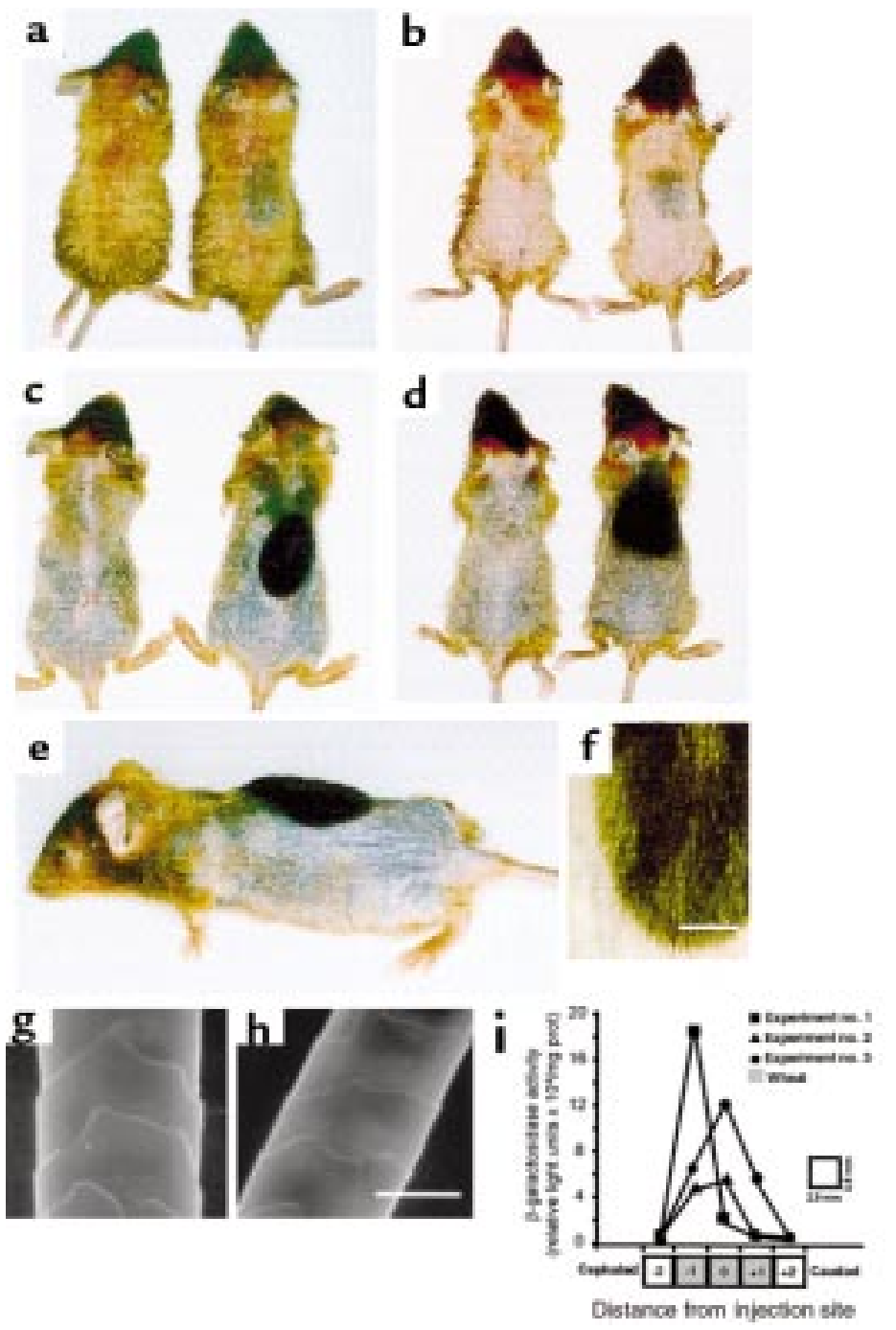

\section{Figure 4}

Hair growth in C57BL/6 mice after intradermal administration of AdShh during first telogen. AdShh, AdNull, or PBS was administered to the dorsal skin of postnatal day 19 C57BL/ 6 mice. Five days after administration, dorsal hair was bleached with blonde hair dye to provide contrast for assessing new growth of the natural black hair of the mice. On day 7 , the dorsal hair was clipped. (a and $\mathbf{b}$ ) Mice at day 7 after administration of AdShh. Shown are 2 example pairs. The injection site in AdNull-treated animals was indistinct (left-side mice), whereas melanogenesis was evident at the site of AdShh injection in AdShh-treated mice (right-side mice). (c and d) Mice at day 14 after administration of AdShh. Shown are 2 example pairs. The injection site in AdNull-treated animals was indistinct (left-side mice). New hair growth is seen (note black color relative to preexisting dyed hair) at the site of AdShh injection (rightside mice). (e) Lateral aspect of day 14 AdShh-treated mouse showing length of hair growth at injection site. ( $f$ ) High magnification of injection site showing new black hair and preexisting blonde-dyed hair. Scale bar: $2 \mathrm{~mm}$. (g) Scanning electron microscopic analysis of normal C57BL/6 mouse hair shaft. (h) Scanning electron microscopic analysis of C57BL/ 6 mouse hair shaft induced by intradermal injection of AdShh (day 14 after vector administration). Scale bar: $10 \mu \mathrm{m}$. (i) Spatial distribution of transgene expression. Adßgal (108 PFU), an E1-E3- Ad vector expressing the Escherichia coli $\beta g a l$ marker gene, was injected intradermally on the dorsal surface of postnatal day 19 C57BL/ 6 mice. On day 0 and on day 2 , a single strip of dorsal skin along the cephalocaudal axis was harvested, divided into 5 equal $2.5-\mathrm{mm}$ segments (see diagram below graph; $0=$ site of infection), and assayed for ßgal activity (data from 3 animals are shown). The gray area corresponds to the size and position of the wheal (7-10 $\mathrm{mm}$ in diameter) formed at the site of injection. Data are expressed as $\beta$ gal activity per $\mathrm{mg}$ protein. The anatomic distribution of the marker gene is similar to that of the melanogenesis and new hair growth observed in $\mathbf{b}$ and $\mathbf{c}$. large, and extension of the follicles deep into the adipose layer of the dermis (Figure 3a). No derangements of the hair follicles were noted in AdShh-injected skin. No abnormal proliferation of basal cells was observed, nor was there evidence of basal cell carcinoma. All hair follicles maintained native orientation along the cephalocaudal axis, indicating that follicle polarity was not altered. The border of the injection site at 7 days after administration of AdShh revealed a sharp contrast in the hair follicle cycle between late anagen in the injection site and early anagen in the adjacent skin (Figure 3b). Quantitative analysis of hair follicle area as a percentage of total skin area (dermal plus epidermal) showed that AdShh induced an increase in follicle area at the site of injection (Figure 3c). In agreement with the histologic data, the expression level for mRNA transcripts of the hair-specific keratin ghHb-1, a molecular marker for anagen hair growth (52), was higher in AdShh-injected animals 7 days after treatment than in naive animals or animals injected with PBS or AdNull (Figure 3d). This is consistent with keratinocyte growth and differentiation induced by administration of AdShh. Melanogenesis was evident in anagen skin as a brown/black area in the matrix of the hair follicle bulb. After injection of AdShh, but not AdNull, the melanin in hair follicle bulbs in unstained paraffin sections was visible, indicating active hair shaft synthesis (Figure 3, e and f). Melanin production requires activity of tyrosinase, an enzyme in the biosynthetic pathway of melanin (51). RNA from skin of AdShh-injected mice 7 days after treatment showed greater levels of tyrosinase mRNA transcripts than did comparable samples from naive, PBS-injected, or AdNull-injected control mice (Figure 3g).

The histologic and molecular evidence indicated that skin was induced to enter anagen when exposed to elevated expression of Shh caused by intradermal administration of AdShh. A corollary to this finding would be that treatment to accelerate the hair follicle cycle's entry to anagen would produce new hair shafts sooner than untreated skin would. To test this hypothesis, dorsal skin of postnatal day 19 C57BL/6 mice were injected with AdShh and compared with naive mice or mice that received PBS or AdNull injections. Five days after injec- 
tion, the hair was dyed with blonde hair dye to mark existing hair. On postnatal day 26 (experimental day 7), mouse hair was carefully clipped and examined for induction of the melanogenesis associated with anagen skin. The dorsal skin of naive mice (not shown), PBSinjected mice (not shown), and AdNull-injected mice (Figure 4, a and b) was of a uniform color, indicating that the dorsal skin appeared similar with respect to the phase of the follicle growth cycle. In contrast, AdShhinjected mouse skin showed a distinct oval of darkened skin in the area of intradermal injection; uninjected skin from the same mice was not affected (Figure 4, a and b; Table 1). On postnatal day 33 (experimental day 14), the area of melanogenesis had produced new hair shafts (note the striking appearance of black hair), whereas the remainder of the dorsal skin had only begun to enter anagen (note darkening of the dorsal skin in Figure 4, c and d; Table 1). In contrast, AdNull-injected animals maintained a uniform hair growth cycle on their dorsal surface (Figure 4, c and d).

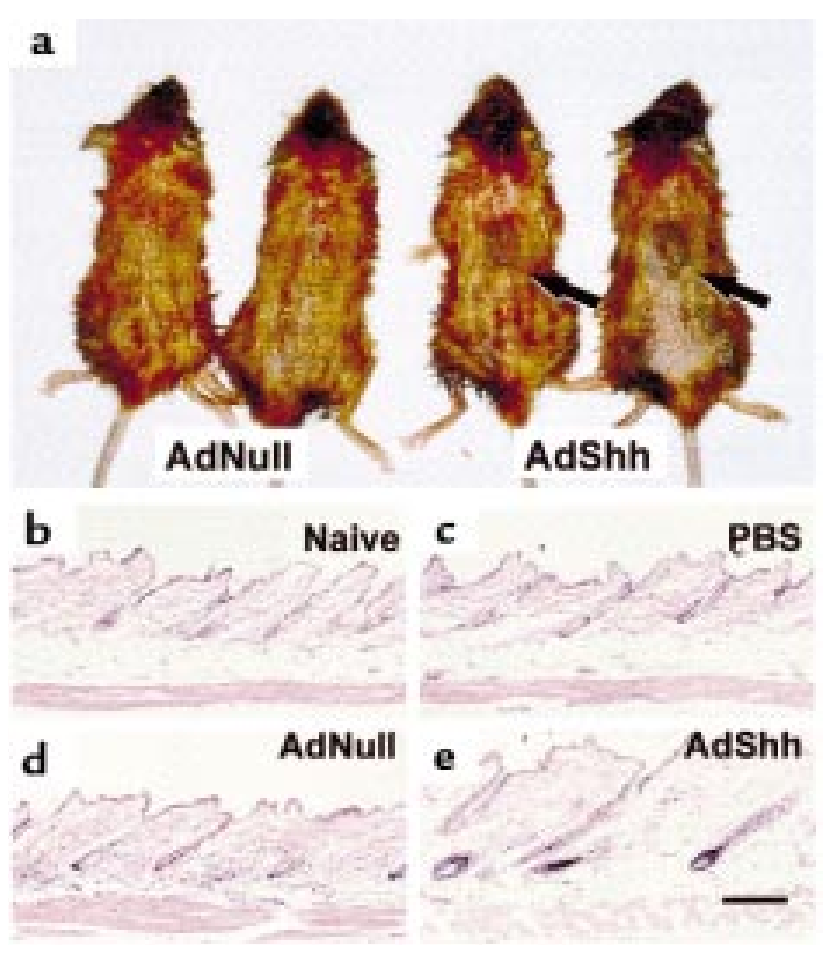

Figure 5

Hair growth in C57BL/ 6 mice after intradermal administration of AdShh during second telogen. AdShh, AdNull, or PBS was administered to the dorsal skin of 8-week-old C57BL/6 mice. Mice were dyed and clipped as described in Figure 3, and were evaluated on experimental day 10 after vector administration for melanogenesis. Dorsal skin was subsequently collected, embedded in paraffin, and sectioned along the cephalocaudal axis. (a) Melanogenesis induced by AdShh. AdShh-treated mice (right pair of mice) but not AdNull-treated mice (left pair of mice) showed melanogenesis at the site of injection 7 days after treatment. (b-e) Histologic evaluation of area of AdShh injection. (b) Naive mouse skin. (c) PBS-injected mouse skin. (d) AdNull-injected mouse skin. (e) AdShh-injected mouse skin. Note the increased thickness and increased area of hair follicles similar to that in Figure 3. Scale bar: $200 \mu \mathrm{m}$.
The newly produced hair shafts in AdShh-treated mice at the gross morphologic level, as well as at the dissecting microscopic and scanning electronic microscopic levels, demonstrated that the newly produced hair shafts had normal hair structures (Figure $4, \mathrm{e}-\mathrm{h})$. The size of the affected area correlated with the size of the dermal wheal formed during intradermal administration of the vector. The hypothesis that transgene expression was limited to the area of the dermal wheal was independently confirmed using an Ad vector expressing the marker gene $\beta$ gal (Adßgal, $10^{8} \mathrm{PFU} / 20 \mu \mathrm{L}$ ), administered to dorsal skin of postnatal day 19 C57BL/6 mice. Two days later, a 12.5 - $\mathrm{mm}$ length of skin $(2.5 \mathrm{~mm}$ wide) was dissected, divided into 5 parts, and analyzed for $\beta$ gal activity. Transgene expression was limited to an area similar in size to the original wheal, with a slight broadening of the expression, indicating that the vector may have spread from the site of injection as the wheal resolved (Figure 4i).

The data indicate that administration of AdShh induced the hair cycle in first skin telogen to enter anagen phase at the site of injection. To demonstrate that AdShh would function in a similar fashion in second telogen, mice were injected with AdShh at 8 weeks of age. Analysis of melanogenesis in mouse skin 10 days after injection showed that AdShh induced melanogenesis, whereas control mice remained in telogen (Figure 5a). Histologic analysis of second telogen skin showed characteristics of anagen at the site of injection of AdShh, but not in control mice (Figure 5, b-e).

Assessment of the AdShh-injected animals $(n=5$ for each treatment) 6 months after administration of the AdShh vector revealed no gross differences compared with AdNull-injected mice (Figure 6a) or other controls (PBS-injected or naive mice, not shown). Histologic examination of dorsal skin 6 months after AdShh administration revealed no abnormalities compared with AdNullinjected, PBS-injected, or naive mice (Figure 6, b-e).

\section{Discussion}

The observations made in this study demonstrate that transient, localized overexpression of Shh in postnatal skin initiates the onset of the anagen growth phase of hair follicles. This conclusion is supported by observations that intradermal administration of an adenovirus gene-transfer vector expressing Shh resulted in enhanced expression of Shh, Ptc, Gli1, hair-specific keratin, and tyrosinase at the injection site. The morphologic observations that AdShh induced increased hair follicle size, increased dermal and epidermal depth, increased melanogenesis, and increased hair growth are all consistent with changes that occur as mouse skin enters anagen. Normal skin histology 6 months after AdShh injection indicated that transient Shh expression in postnatal skin did not induce pathologic abnormalities such as those associated with constitutive overexpression of Shh or the Wnt signaling pathway throughout development $(34,36)$. Together with the accumulated knowledge of 


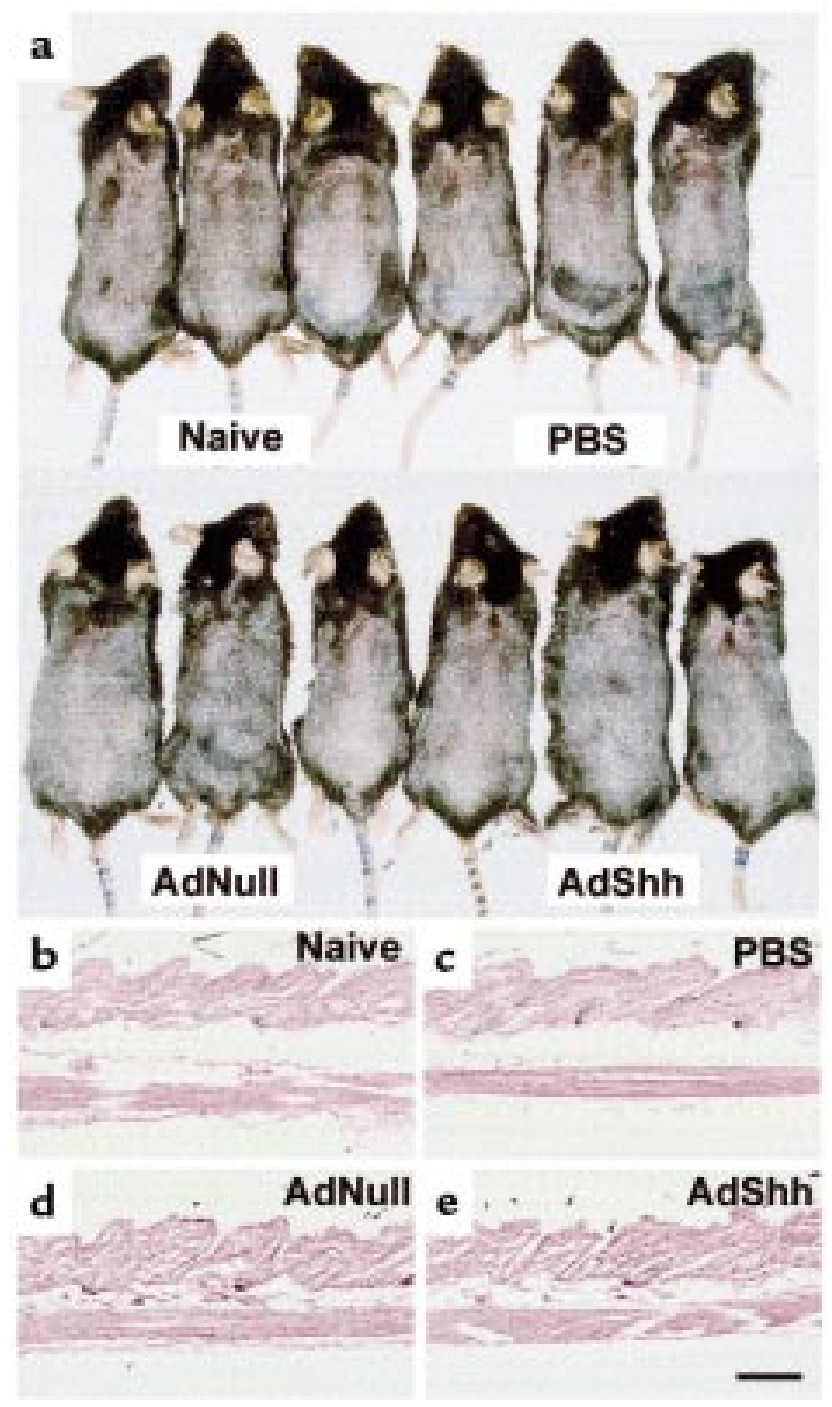

Figure 6

Macroscopic and histologic assessment of AdShh-injected mice 6 months after administration of AdShh. (a) Macroscopic assessment of PBS-, AdNull-, and AdShh-injected mice. For long-term studies, mice were not exposed to hair dye, but were clipped once at the time of vector administration. No differences were noted among groups. (b-e) Histologic assessment. Shown are representative sections from skin of a naive mouse and of PBS-, AdNull-, and AdShh-injected mice 6 months after administration. No abnormalities were observed at the injection site at this time. Three mice were examined for each treatment. Scale bar: $200 \mu \mathrm{m}$.

hair follicle differentiation $(1,2)$, our data are consistent with the concept that transient upregulation of Shh activity in postnatal skin functions as a biologic switch that induces resting telogen follicles to enter the anagen growth phase, with consequent hair growth.

In situ hybridization was performed using mouse skin 3 days after administration of the vector, a timepoint at which the Ad vector-mediated Shh is expressed in excess over endogenous Shh. Strong positive signals were noted in diverse cells types, similar to reports of adenovirus-mediated transgene expression in mouse skin (42). Many hair follicles did not appear to express vector-derived Shh, but Shh-expressing cells were observed in the vicinity of most hair follicles. Given the density of positive in situ hybridization staining in the sections, it is likely that hair follicles were exposed to additional Shh-secreting cells present in tissue adjacent to the section subjected to in situ analysis. Histologic examination of AdShh-injected skin demonstrated that hair follicles uniformly entered anagen. Uniform induction of anagen suggested that Shh expression from vector-derived Shh or a combination of vector-derived Shh and induced expression of endogenous Shh was capable of inducing anagen throughout the area of injection. From the available data, we cannot identify which specific cell type(s) in hair follicles must express Shh to initiate induction of anagen. An alternative interpretation is that vector-derived Shh induced endogenous Shh, either directly or through stimulation of a positivefeedback loop, leading to expression of endogenous Shh in cells that were physiologically relevant to anagen induction. This explanation is consistent with the suggestion in the literature that communication between epithelial and mesenchymal structures modulates hair follicle development and cycling $(1,19,39)$. In examining either of these alternatives, it is important to note that whereas vector-derived, ectopic Shh expression was sufficient to induce anagen in the injection area, spread of anagen among follicles was not likely to depend solelyon endogenous Shh. If endogenous Shh were responsible for anagen spread within the injection site, then it should also be capable of generating a wave of anagen in the adjacent tissue at the borders of the injection site. Histologic analysis showed that a sharp border existed between the injection site (where the hair follicle cycle was altered) and adjacent skin (where the hair follicle cycle was not altered). Anagen was not observed to propagate outside the injection site.

To be characterized as a biologic switch, Shh should be able to induce a change that modifies the natural progression of the hair cycle. Three pieces of experimental evidence point to the ability of Shh to induce the anagen phase of hair growth. First, endogenous Shh is normally expressed in early anagen, consistent with a role in triggering later changes in anagen. The data show that Shh is expressed early in anagen (postnatal day 26), but has decreased expression later in anagen (postnatal day 33). Second, using a variety of molecular and morphologic criteria, the data indicate that AdShh administration during the first catagen/first telogen transition (day 19) leads to early initiation of second anagen phase at the injection site. Northern analysis and in situ hybridization data indicate that the AdShh vector drives rapid expression of the Shh message after infection, with expression occurring before the natural onset of early anagen; therefore exogenous Shh expression is likely to initiate the onset of anagen rather than accelerating anagen that has already been initiated. This concept was further sup- 
ported by experiments with AdShh administration to 8 -week-old mice in the second cycle of telogen phase. The considerably longer duration (5-6 weeks) of second telogen phase before onset of third anagen phase provides an unambiguous demonstration of anagen induction by AdShh.

To date, a number of growth factors have been implicated in hair follicle growth and progression through the hair cycle $(7,8)$, but the key signaling molecules responsible for unleashing the coordinated cascade of factors mediating the transition from telogen to anagen remain to be identified. The relevance of Shh to hair development has been suggested in both gain- and lossof-function models wherein modified Shh expression exists throughout embryonic development. In a loss-offunction model, fetal skin from mouse Shb homozygous mutants $\left(\mathrm{Shb}^{--}\right)$fails to show normal epithelial invaginations during hair follicle development $(19,39)$. When skin from $\mathrm{Shb}^{-1-}$ mice is transplanted into a nude mouse host, the $S h h^{-/-}$skin fails to differentiate properly, and instead forms hyperproliferative follicle-like structures that do not produce mature hair shafts $(19,39)$. In contrast, gain-of-function models in embryonic development show that forced expression of Shh or of genes related to Shh signaling in the skin induces abnormal epidermal cell proliferation and hair follicle orientation $(21,28,34-36,38)$. Despite the fact that persistent activity of the Shh pathway in skin throughout embryonic development results in hyperproliferation of skin basal cells (36), transplantation of Shb transgenic embryo skin into adult scid mice leads to partial differentiation of skin and development of structures with some characteristics of hair follicles, suggesting that the consequences of enhanced expression of Shh in postnatal skin can be controlled. Similarly, the stage of development at which Shh overexpression is induced in chick feather buds can affect the severity of abnormal bud formation (28). Consistent with this concept, the transient enhanced expression of the Shb gene in postnatal skin observed in this study resulted in controlled acceleration of the onset of follicle development and subsequent hair growth, without excessive proliferation of basal cell keratinocytes. The data from this study, compared with constitutive expression models $(21,28,35$, 36,38 ), suggest that appropriate windows for the extent and duration of Shh expression appropriate for the development of normal structures can be identified, and that these levels may differ depending on the stage of tissue development (prenatal vs. postnatal) and the target tissue (e.g., hair follicle, lung, brain).

The ability of AdShh to accelerate the induction of anagen in a background of telogen skin invites the interesting speculation that transient expression of Shh could activate the hair growth cycle in disease conditions where the extent of these functions has been reduced. In this regard, the observations in this study may be relevant to a new approach to hair loss therapy, in which a gene-transfer vector provides localized, transient overexpression of activity of the Shh pathway, resulting in acceleration of hair follicle cycling to anagen, and thus hair growth. For example, such a strategy may be beneficial in treating some forms of alopecia associated with chemotherapy (7), in the same fashion as hematologic growth factors are used to accelerate the development of hematologic progenitors (53). These observations also suggest that the expression of other genes in the Shh pathway, such as Ptc and Gli1, could also be targets for therapeutic induction or inhibition of hair growth, using gene-transfer, antisense, or small-molecule therapeutic strategies. Whether modification of the expression of the Shh pathway is applicable to common androgenic alopecia (male pattern baldness) will have to await further study.

\section{Acknowledgments}

We thank A.P. McMahon of Harvard University for the murine Shh cDNA; M.P. Scott of Stanford University for the murine Ptc cDNA; B. Vogelstein of Johns Hopkins University for the human Gli1 cDNA; A. Panteleyev of Columbia University and J. Reed of Cornell University for help in evaluating the histologic sections; R. Singh for help with the Adßgal studies; B. Ferris for assistance with the in situ hybridization studies; the Electron Microscopy Core Facility of the Weill Medical College of Cornell University; and N. Mohamed for help in preparing this manuscript. These studies were supported in part by the Will Rogers Memorial Fund and GenVec Inc.

1. Hardy, M.H. 1992. The secret life of the hair follicle. Trends Genet. 8:55-61.

2. Oro, A.E., and Scott, M.P. 1998. Splitting hairs: dissecting roles of signaling systems in epidermal development. Cell. 95:575-578.

3. Sengel, P. 1976. Morphogenesis of skin. Cambridge University Press. Cambridge, United Kingdom. pp. 20-29.

4. Wessells, N.K., and Rommens, J.M. 1965. Nonproliferation in dermal condensations of mouse vibrissae and pelage hairs. Dev. Biol. 12:419-433.

5. Bertolino, A.P., Klein, L.M., and Freedberg, I.M. 1993. Biology of hair follicles. In Dermatology in general medicine. T.B. Fitzpatrick, A.Z. Eisen, K. Wolff, I.M. Freedberg, and K.F. Austen, editors. McGraw-Hill Inc. New York, NY. 289-293.

6. Kligman, A.M. 1959. The human hair cycle. J. Invest. Dermatol. 33:307-316.

7. Danilenko, D.M., Ring, B.D., and Pierce, G.F. 1996. Growth factors and cytokines in hair follicle development and cycling: recent insights from animal models and the potentials for clinical therapy. Mol. Med. Today. 2:460-467.

8. Stenn, K.S., et al. 1996. Hair follicle growth controls. Dermatol. Clin. 14:543-558.

9. Bellusci, S., et al. 1997. Involvement of Sonic hedgehog (Shh) in mouse embryonic lung growth and morphogenesis. Development. 124:53-63.

10. Bitgood, M.J., and McMahon, A.P. 1995. Hedgehog and BMP genes are coexpressed at many diverse sites of cell-cell interaction in the mouse embryo. Dev. Biol. 172:126-138.

11. Chiang, C., et al. 1996. Cyclopia and defective axial patterning in mice lacking Sonic hedgehog gene function. Nature. 383:407-413.

12. Echelard, Y., et al. 1993. Sonic hedgehog, a member of a family of putative signaling molecules, is implicated in the regulation of CNS polarity. Cell. 75:1417-1430.

13. Ingham, P.W. 1998. Transducing hedgehog: the story so far. EMBOJ. 17:3505-3511.

14. Krauss, S., Concordet, J.P., and Ingham, P.W. 1993. A functionally conserved homolog of the Drosophila segment polarity gene hh is expressed in tissues with polarizing activity in zebrafish embryos. Cell. 75:1431-1444.

15. Litingtung, Y., Lei, L., Westphal, H., and Chiang, C. 1998. Sonic hedgehog is essential to foregut development. Nat. Genet. 20:58-61.

16. Marigo, V., et al. 1995. Cloning, expression, and chromosomal location of SHH and IHH: two human homologues of the Drosophila segment polarity gene hedgehog. Genomics. 28:44-51. 
17. Nusslein-Volhard, C., and Wieschaus, E. 1980. Mutations affecting segment number and polarity in Drosophila. Nature. 287:795-801.

18. Riddle, R.D., Johnson, R.L., Laufer, E., and Tabin, C. 1993. Sonic hedgehog mediates the polarizing activity of the ZPA. Cell. 75:1401-1416.

19. St.-Jacques, B., et al. 1998. Sonic hedgehog signaling is essential for hair development. Curr. Biol. 8:1058-1068.

20. Iseki, S., et al. 1996. Sonic hedgehog is expressed in epithelial cells during development of whisker, hair, and tooth. Biochem. Biophys. Res. Commun. 218:688-693.

21. Dahmane, N., et al. 1997. Activation of the transcription factor Gli1 and the Sonic hedgehog signalling pathway in skin tumours. Nature. 389:876-881.

22. Goodrich, L.V., Johnson, R.L., Milenkovic, L., McMahon, J.A., and Scott, M.P. 1996. Conservation of the hedgehog/patched signaling pathway from flies to mice: induction of a mouse patched gene by Hedgehog. Genes Dev. 10:301-312.

23. Hammerschmidt, M., Brook, A., and McMahon, A.P. 1997. The world according to hedgehog. Trends Genet. 13:14-21.

24. Hui, C.C., and Joyner, A.L. 1993. A mouse model of Greig cephalopolysyndactyly syndrome: the extra-toes mutation contains an intragenic deletion of the Gli3 gene. Nat. Genet. 3:241-246.

25. Marcelle, C., Stark, M.R., and Bronner-Fraser, M. 1997. Coordinate actions of BMPs, Wnts, Shh and noggin mediate patterning of the dorsal somite. Development. 124:3955-3963.

26. Matise, M.P., Epstein, D.J., Park, H.L., Platt, K.A., and Joyner, A.L. 1998. Gli2 is required for induction of floor plate and adjacent cells, but not most ventral neurons in the mouse central nervous system. Development. 125:2759-2770.

27. Mo, R., et al. 1997. Specific and redundant functions of Gli2 and Gli3 zinc finger genes in skeletal patterning and development. Development. 124:113-123.

28. Morgan, B.A., Orkin, R.W., Noramly, S., and Perez, A. 1998. Stage-specific effects of sonic hedgehog expression in the epidermis. Dev. Biol. 201:1-12.

29. Motoyama, J., Takabatake, T., Takeshima, K., and Hui, C. 1998. Ptch2, a second mouse Patched gene is co-expressed with Sonic hedgehog. Nat. Genet. 18:104-106.

30. Quirk, J., et al. 1997. The smoothened gene and hedgehog signal transduction in Drosophila and vertebrate development. Cold Spring Harb. Symp. Quant. Biol. 26:217-226.

31. Sasaki, H., Hui, C., Nakafuku, M., and Kondoh, H. 1997. A binding site for Gli proteins is essential for HNF-3beta floor plate enhancer activity in transgenics and can respond to Shh in vitro. Development. 124:1313-1322.

32. Stone, D.M., et al. 1996. The tumour-suppressor gene patched encodes a candidate receptor for Sonic hedgehog. Nature. 384:129-134

33. Yoon, J.W., et al. 1998. GLI activates transcription through a herpes simplex viral protein 16-like activation domain. J. Biol. Chem. 273:3496-3501.

34. Gat, U., DasGupta, R., Degenstein, L., and Fuchs, E. 1998. De novo hair follicle morphogenesis and hair tumors in mice expressing a truncated beta-catenin in skin. Cell. 95:605-614.
35. Fan, H., Oro, A.E., Scott, M.P., and Khavari, P.A. 1997. Induction of basal cell carcinoma features in transgenic human skin expressing Sonic Hedgehog. Nat. Med. 3:788-792.

36. Oro, A.E., et al. 1997. Basal cell carcinomas in mice overexpressing sonic hedgehog. Science. 276:817-821.

37. Ting-Berreth, S.A., and Chuong, C.M. 1996. Sonic Hedgehog in feather morphogenesis: induction of mesenchymal condensation and association with cell death. Dev. Dyn. 207:157-170.

38. Xie, J., et al. 1998. Activating Smoothened mutations in sporadic basalcell carcinoma. Nature. 391:90-92.

39. Chiang, C., et al. 1999. Essential role for Sonic hedgehog during hair follicle morphogenesis. Dev. Biol. 205:1-9.

40. Kovesdi, I., Brough, D.E., Bruder, J.T., and Wickham, T.J. 1997. Adenoviral vectors for gene transfer. Curr. Opin. Biotechnol. 8:583-589.

41. Wilson, J.M. 1996. Adenoviruses as gene-delivery vehicles. N. Engl. J. Med. 334:1185-1187.

42. Setoguchi, Y., Jaffe, H.A., Danel, C., and Crystal, R.G. 1994. Ex vivo and in vivo gene transfer to the skin using replication-deficient recombinant adenovirus vectors. J. Invest. Dermatol. 102:415-421.

43. Rosenfeld, M.A., et al. 1992. In vivo transfer of the human cystic fibrosis transmembrane conductance regulator gene to the airway epithelium. Cell. 68:143-155.

44. Hersh, J., Crystal, R.G., and Bewig, B. 1995. Modulation of gene expression after replication-deficient, recombinant adenovirus-mediated gene transfer by the product of a second adenovirus vector. Gene Ther. 2:124-131.

45. Crystal, R.G., et al. 1994. Administration of an adenovirus containing the human CFTR cDNA to the respiratory tract of individuals with cystic fibrosis. Nat. Genet. 8:42-51.

46. Chase, H.B. 1954. Growth of the hair. Physiol. Rev. 34:113-126.

47. du Cros, D.L. 1993. Fibroblast growth factor influences the development and cycling of murine hair follicles. Dev. Biol. 156:444-453.

48. Chen, W.S., et al. 1994. Disruption of the HNF-4 gene, expressed in visceral endoderm, leads to cell death in embryonic ectoderm and impaired gastrulation of mouse embryos. Genes Dev. 8:2466-2477.

49. Manova, K., et al. 1998. Apoptosis in mouse embryos: elevated levels in pregastrulae and in the distal anterior region of gastrulae of normal and mutant mice. Dev. Dyn. 213:293-308

50. Wang, X., et al. 1996. A candidate gene for the amnionless gastrulation stage mouse mutation encodes a TRAF-related protein. Dev. Biol. 177:274-290

51. Slominski, A., Paus, R., and Costantino, R. 1991. Differential expression and activity of melanogenesis-related proteins during induced hair growth in mice. J. Invest. Dermatol. 96:172-179.

52. Bowden, P.E., et al. 1998. Characterization and chromosomal localization of human hair-specific keratin genes and comparative expression during the hair growth cycle. J. Invest. Dermatol. 110:158-164.

53. Welte, K., Gabrilove, J., Bronchud, M.H., Platzer, E., and Morstyn, G. 1996. Filgrastim (r-metHuG-CSF): the first 10 years. Blood. 88:1907-1929. 